\title{
Preextubation Fentanyl on Hemodynamics \& Recovery Profile, A Prospective Randomized Controlled Study
}

Ahmed salman*, Ashraf Mohammed** ,Sahar Abdelhalem*, and Amel Abo Elela**

* National Cancer Inistitute, Cairo University, Cairo, Egypt

** Faculty of Medicine, Cairo University, Cairo, Egypt

Keywords: Preextubation, Fentanyl, Heamodynamics, Extubation Time.

\section{Background and rationale}

Tracheal extubation, as well as intubation provoke stressful responses, including tachycardia and hypertension, extubation also provoke airways reflexes causing cough or strain, which are known to increase blood pressure and heart rate. These hemodynamic changes during extubation and emergence from anesthesia may cause dangerous cardiovascular complications. Fentanyl has been used extensively to maintain perioperative hemodynamic stability. This study was carried out to evaluate the effect of fentanyl on cardiovascular responses and recovery profile to tracheal extubation.

\section{Methodology}

We studied 126 patients with controlled hypertension, received chemotherapy undergoing mastectomy operation for breast cancer. The patients divided into 3 groups $(n=42)$ were the first group received $2 \mathrm{mic} / \mathrm{kg}$ fentanyl at the end of anesthesia, the second group received $1 \mathrm{mic} / \mathrm{kg}$, while the third group received $10 \mathrm{ml}$ saline as placebo at the same time. Hemodynamics (Mean blood pressure, Heart rate), Extubation time, Behavioral status (Sedation, Alertness or Agitation) and Complication after extubation (Desaturation, Vomiting, Coughing, Laryngospasm and Shivering) were recorded.

\section{Results and Discussion}

In patients with controlled hypertension, received chemotherapy undergoing mastectomy operation for breast cancer; intravenous fentanyl $1 \mathrm{mic} / \mathrm{kg}$ given at the end of anesthesia blunts cardiovascular responses to tracheal extubation and emergence from anesthesia. This does not lead to respiratory depression or prolonged recovery. The use of 2 $\mathrm{mic} / \mathrm{kg}$ fentanyl at the same time provides more control on the heart rate than $1 \mathrm{mic} / \mathrm{kg}$ but with delay in the extubation time.

\section{Conclusion}

Preextubation intravenous fentanyl $1 \mathrm{mic} / \mathrm{kg}$ given at the end of anesthesia is a simple, effective, and practical method in obliterating cardiovascular responses to tracheal extubation and emergence from anesthesia with no respiratory depression or prolonged recovery. 